\title{
Perioperative Considerations for Acute Appendicitis in Patients With Coronavirus Infection: Two Cases Report
}

\author{
In-Kyeong Kim ${ }^{1}$, Seung-jin Kwag ${ }^{2}$, Han-Gil Kim², Young-Tae Ju², Seung-Jun Lee ${ }^{3}$ Tae-Jin Park ${ }^{1}$, \\ Sang-Ho Jeong ${ }^{1}$, Eun-Jung Jung ${ }^{1}$, Jin-Kwon Lee ${ }^{1}$ \\ ${ }^{1}$ Department of Surgery, Gyeongsang National University Changwon Hospital, Changwon; ${ }^{2}$ Department of Surgery, Gyeongsang National \\ University Hospital, Jinju; ${ }^{3}$ Department of Convergence Medical Science, Gyeongsang National University, Jinju, Korea
}

\begin{abstract}
We report considerations related with surgery through 2 cases of acute appendicitis (AA) with coronavirus disease 2019 (COVID-19) infection. In November and December 2020, AA occurred in 2 patients with COVID-19, who underwent emergency surgery. In case 1, an 84-year-old woman was asymptomatic and diagnosed with AA on the 20th day of infection. She was discharged after surgery without complication. In contrast, case 2 was that a 69 -year-old man with pneumonia was treated with antibiotics, steroids, and remdesivir. After surgery, he was hospitalized for a long duration due to persistent pneumonia and wound complications. We should perform in well-established negative pressure operating rooms, personal protective equipment, and protocols. Since the physical examination and blood tests were limited, image examination like computed tomography scan should be considered if AA is suspected. If pneumonia is accompanied before surgery, pneumonia may worsen after surgery, or complications such as wound infection may occur
\end{abstract}

\section{Keywords: Appendicitis; COVID-19; Diagnosis; Perioperative care}

\section{INTRODUCTION}

Coronavirus disease 2019 (COVID-19) has spread worldwide since December 2019, and the World Health Organization declared a pandemic on March 11, 2019 [1]. As a result, the health care system is facing a crisis. Fear of self-isolation has reduced access to hospitals, delaying diagnosis and timely use of medical facilities. In cases requiring emergency surgery, complications are on the rise. Acute appendicitis is the most common abdominal disease requiring surgery [2] and is prevalent in the COVID-19 pandemic. Therefore, we report preoperative evaluation, intraoperative management, postoperative treatment, and complications

Received: Jul 27, 2021 - Revised: Aug 23, 2021 - Accepted: Sep 6, 2021

Correspondence to: Jin-Kwon Lee, M.D.

Department of Surgery, Gyeongsang National University Changwon

Hospital, 11 Samjeongja-ro, Seongsan-gu, Changwon 51472, Korea

Tel: +82-55-214-3774, Fax: +82-55-214-3251

E-mail: gsbigcap@gmail.com

ORCID: https://orcid.org/0000-0001-6113-7252

(C) 2021 The Korean Society of Coloproctology

This is an open-access article distributed under the terms of the Creative Commons Attribution NonCommercial License (https://creativecommons.org/licenses/by-nc/4.0) which permits unrestricted non-

commercial use, distribution, and reproduction in any medium, provided the original work is properly cited. through 2 cases of acute appendicitis managed surgically with COVID-19 infection.

\section{CASE REPORTS}

This report was approved by the Institutional Review Board of Gyeongsang National University Changwon Hospital (No. 202106-033) and the patient provided informed consent for the publication of the report and accompanying images.

\section{General principle of operation in COVID-19 infected patients}

Surgery was performed in a negative pressure operating room maintained at $-0.25 \mathrm{mmAq}$ and turned on at least 30 minutes before surgery. Patient wearing level D personnel protective gear was transported by a negative pressure cart via designated route. Anesthesiologists wore respiratory protective equipment (powered air-purifying respirator, PAPR) and intubated the patient with an endotracheal tube under general anesthesia using tracheal intubation shield. Surgeons and nurses who participated in surgery wore double gowns, N95 masks, goggles, neck protection, nurse cap, overshoes, double gloves, and PAPR, as necessary (Fig. 1). 


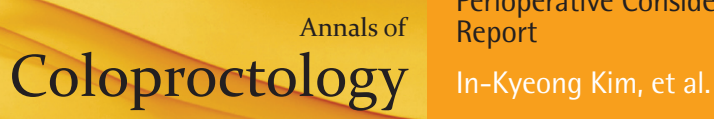

The operation was performed laparoscopy and thoroughly equipped with personal protective equipment (Fig. 2). The instruments needed for surgery were provided by placing a cart near the entrance of the front room. Samples were placed in a zipper bag for emergency blood tests, sterilized at 1,000 ppm, and disinfected externally. They were transferred to the pathology department in a dedicated container.

After surgery, the patient was extubated in the negative pressure operating room and transferred to the negative pressure ward with a disposable mask on a negative pressure cart. Surgical instruments were prepared using disposable instruments and transferred to the washing room to prevent contamination of the sur-

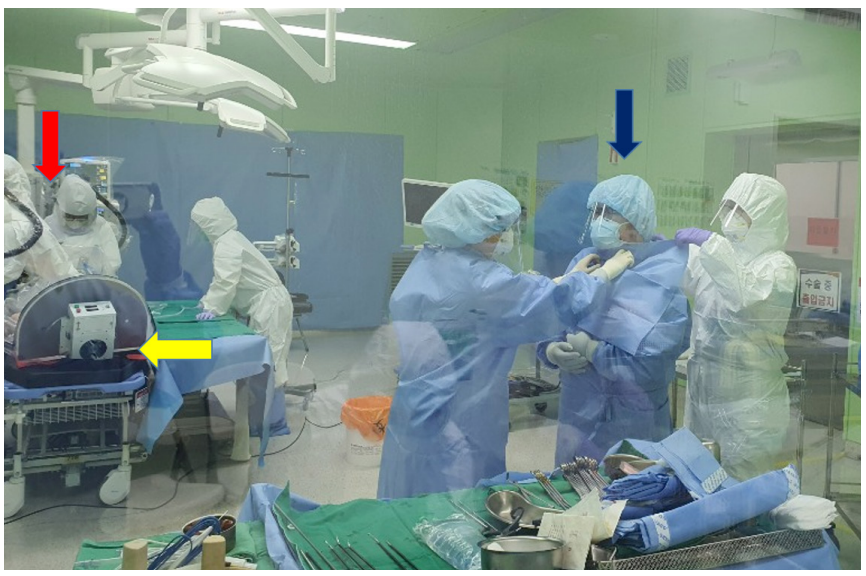

Fig. 1. Preparation for surgery. Red arrow show anesthesiologist and nurses wore respiratory protective equipment (powered air-purifying respirator). Yellow arrow show transported a patient with coronavirus disease 2019 (COVID-19) infection by a negative pressure cart. A surgeon (blue arrow) is wearing double gowns, N95 masks, goggles, neck protection, nurse cap, neck protection, nurse cap, double gloves, and overshoes.

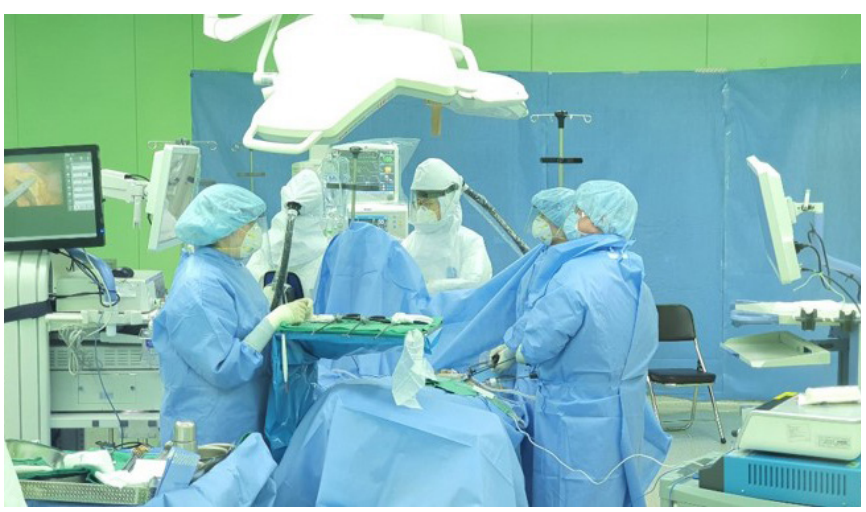

Fig. 2. The surgeon, nurses, and anesthesiologist wore the protective equipment each other during a laparoscopic appendectomy in a negative pressure operating room maintained at $-0.25 \mathrm{mmAg}$. rounding environment. All appliances and the environment were sterilized by cleaning thoroughly with a moist disposable towel using a disinfectant. The environmental disinfection range involved all areas of patient movement including all areas used for the negative pressure surgery. As a preventative measure, ventilation of the environment was performed for more than 4 hours after environmental disinfection.

\section{Case 1}

An 84-year-old female with dementia was diagnosed with asymptomatic COVID-19 infection on November 4, 2020. On the 20th day of hospitalization in the Busan Medical Center, fever and lower abdominal pain developed, and abdominal computed tomography (CT) was performed. It revealed appendiceal lumen dilatation and wall thickening with fat stranding, leading to the diagnosis of acute appendicitis (Fig. 3). Blood tests were unremarkable except for elevated levels of C-reactive protein (CRP; $61.30 \mathrm{mg} / \mathrm{L}$ ). White blood cell (WBC) was within normal limits $(4,670 / \mu \mathrm{L})$. Chest CT showed unremarkable except for the evidence of cardiac hypertrophy. Therefore, emergency surgery was performed with a laparoscopic 3-port method. Intraoperative considerations was reviewed above in the section on 'General principles of surgery for COVID-19 infected patients.'

It took 10 minutes to transfer the patient to the operating room, 20 minutes to prepare for anesthesia, and 30 minutes to move the negative pressure intensive care unit after the end of the anesthesia. The operation time from skin incision to suture was $35 \mathrm{~min}$ utes, and anesthesia time was 65 minutes. No complications occurred during surgery. After surgery, the patient drank water on the 2nd day and soft food on the 4th day. There were no postoperative complications. On the 5th and 6th days, the COVID-19 polymerase chain reaction (PCR) test showed negative results, and the patient was released from quarantine. On the 7th day, the wound stitch was removed and the patient was discharged.
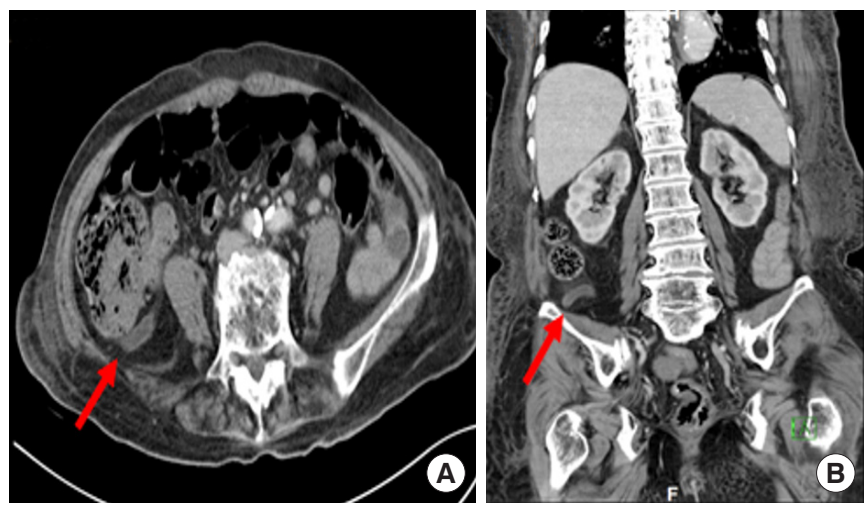

Fig. 3. Abdominal computed tomography findings of case 1 showed that appendiceal lumen dilatation and wall thickening with fat stranding (red arrows). There are no perforation or intraabdominal abscess. (A) Axial view. (B) Coronal view. 

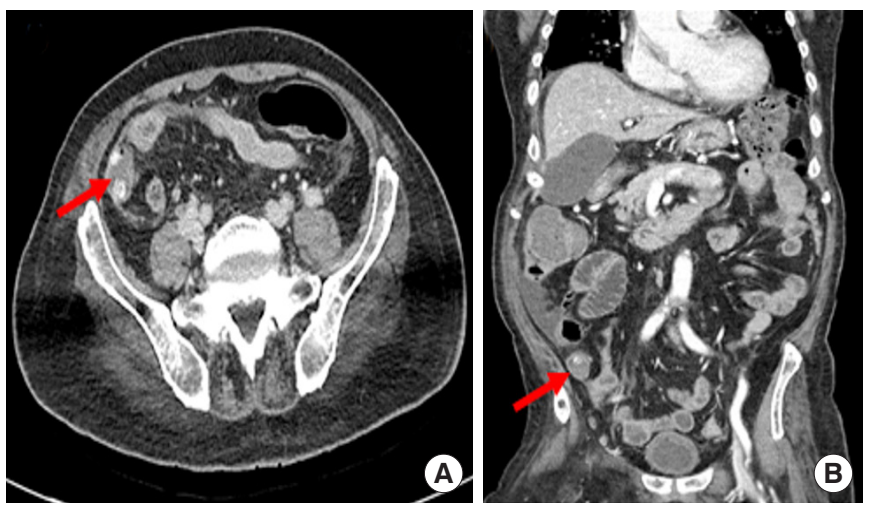

Fig. 4. Abdominal computed tomography findings of case 2 showed that acute appendicitis with perforation and scanty fluid collection (red arrows). (A) Axial view. (B) Coronal view.

\section{Case 2}

A 69-year-old male with Alzheimer dementia was hospitalized at a local hospital in Eumseong, Korea. After fever up to $39.0^{\circ} \mathrm{C}$, COVID-19 was confirmed on December 22, 2020. As worsened pneumonia, he was admitted to the negative pressure intensive care unit of our medical center. A high-flow nasal cannula optic flow of $40 \%$ at $40 \mathrm{~L}$ was applied, and $100 \mathrm{mg}$ of remdesivir was administered via the intravenous route for 4 days. In addition, antibiotics such as levofloxacin and teicoplanin, and $40 \mathrm{mg}$ of dexamethasone were administered intravenously.

Leukocytosis persisted from the initial value of $12,000 / \mathrm{mL}$ to $20,480 / \mathrm{mL}$ and declined in the CRP levels from $53.1 \mathrm{mg} / \mathrm{L}$ on day 1 of hospitalization to $5.1 \mathrm{mg} / \mathrm{L}$ on day 7 . On the 11th day of hospitalization, the patient presented with fever, tachycardia (heart rate $>160$ beats per minute), and lower abdominal pain. CRP levels increased to $132.4 \mathrm{mg} / \mathrm{L}$, and WBC count decreased to 7,300/mL. Abdominal CT revealed appendicitis with perforation (Fig. 4). Chest CT showed pneumonia with extensive peribronchovascular ground-glass opacities and consolidation in both lungs.

A general laparoscopic 3-port surgery was performed under a personal protective device and negative pressure facility similar to case 1. Laparoscopic cecectomy with intraabdominal drainage was performed due to perforation and spread of stools in the abdominal cavity (Fig. 5). It took 18 minutes to transfer the patient to the operating room, 15 minutes to prepare for anesthesia, and 15 minutes to move the negative pressure intensive care unit after the end of anesthesia. The operation time from skin incision to suture was 55 minutes and anesthesia time was 80 minutes.

As pneumonia persisted after surgery, ventilation care was maintained in the negative pressure intensive care unit. Intraperitoneal findings were improved; but with difficulty in oral dietary intake due to pneumonia, the patient could feed through the Levin tube on the 2 nd postoperative day. Intraperitoneal drainage tube was removed on the 8th day. To treat persistent pneumonia,
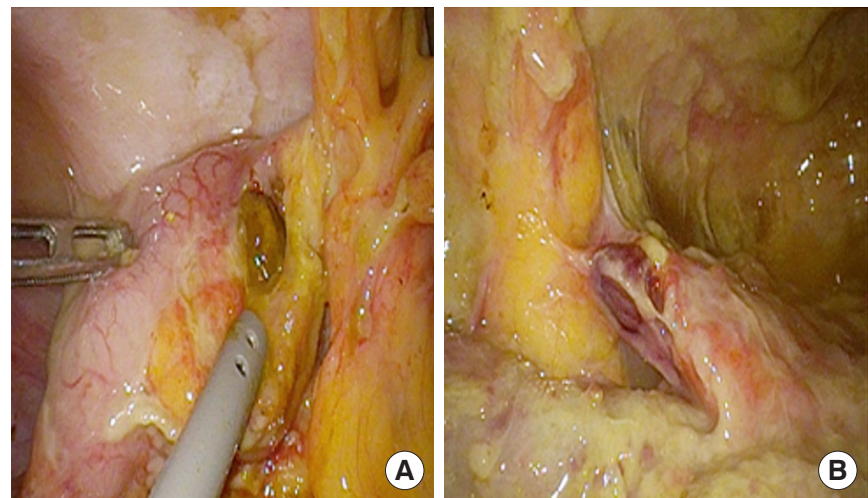

Fig. 5. Surgical findings showed that there was severe inflammation and appendiceal perforation with diffuse turbid exudates. (A) Bowel content with perforation. (B) Necrosis of appendix with turbid exudates.

percutaneous dilatational tracheostomy was performed and administration of dexamethasone and antibiotics continue. On the 23rd postoperative day, a wound of the trocar insertion site was separated, and a simple suture was performed. Pneumonia improved after intensive care and a negative PCR result for COVID-19 was obtained on the 28th postoperative day. The patient was transferred to local hospital.

\section{DISCUSSION}

Acute appendicitis has occurred frequently even before the COVID-19 pandemic. However, due to fear of visiting the hospital, diagnosis and treatment of acute appendicitis tend to be delayed. Yang et al. [3] reported that the average time between the onset of symptoms and hospitalization due to acute appendicitis was 24.57 hours before and 37.92 hours during the COVID-19 pandemic. Also, when comparing the severity of the disease, the incidence of suppurative appendicitis was increased by $86.6 \%$ compared to $76.0 \%$ before the pandemic, and the incidence of complex appendicitis increased from $19.4 \%$ to $35.8 \%$. Thus, acute complicated appendicitis has increased due to delayed hospital visits owing to the COVID-19 pandemic.

Bajomo et al. [4] compared the clinical course of patients with acute appendicitis before and during the COVID-19 pandemic; the mean CRP levels increased significantly from 54.0 to $103 \mathrm{mg} / \mathrm{L}$. On the other hand, WBC counts were slightly higher during the pandemic, but the difference was not significant. Compared to our review, the CRP levels rose to $132.4 \mathrm{mg} / \mathrm{L}$ in case 2, but 61.30 $\mathrm{mg} / \mathrm{L}$ in case 1 which was similar to the average value before the pandemic. It showed blood tests were insufficient to make a differential diagnosis [4]. Therefore, we recommend that imaging studies such as abdominal CT be actively used to diagnose acute appendicitis. Brown [5] reported the efficacy of abdominal CT was $87.5 \%$ for the diagnosis of acute appendicitis during the COVID-19 pandemic compared to $69.8 \%$ before it. Abdominal CT 
has a high sensitivity (97\%) and specificity (93\%) for the diagnosis of appendicitis. Somers et al. [6] reported that abdominal CT was widely used for diagnosis, and the rate of uncomplicated appendicitis increased from $54.05 \%$ to $69.64 \%$ in the pandemic. It also showed a decrease in histologic severity and overall complication. This would be able to reduce the risk of perioperative viral transmission [6]. As the incidence of delayed diagnosis and complicated appendicitis has increased in the pandemic, abdominal CT should be considered for rapid and accurate diagnosis and treatment.

Despite an increased rate of complication, there has been no change in treatment before and during the COVID-19 pandemic. According to the World Society of Emergency Surgery guideline published in 2021, uncomplicated appendicitis can be treated with antibiotics and conservative treatment without surgery during the COVID-19 pandemic. For complicated appendicitis such as appendicular abscess, percutaneous drainage is first attempted; in cases of perforated appendicitis, laparoscopy is performed if appropriate equipment is available [7]. Therefore, we performed laparoscopic surgery with a general 3-port in our case of perforation without abscess.

There is no evidence suggesting transmission of COVID-19 through surgical smoke. However, Mowbray et al. [8] report the possibility of a theoretical risk of virus transmission such as coronavirus. There is a theoretical risk of virus transmission because it is similar in structure to viruses which are already known to be transmitted through surgical smoke, such as Corynebacterium, human papillomavirus, poliovirus, human immunodeficiency virus, and hepatitis B virus. Therefore, it is recommended to perform laparoscopic surgery rather than open surgery with negative pressure operating room set-up, patient movement, and operation theater equipment when operating on COVID-19 infected patients [8]. So, our medical center also recognizes the increased need for emergency surgery for such patients and reflects the guidelines of infection control laid out by the Center for Disease Control and Prevention through the cooperation of the surgical, anesthesiology, anesthesiologists, and nursing departments. Therefore, the manual was prepared in advance, and the simulation was conducted thoroughly.

Postoperative complications are important to be considered in patients infected with COVID-19. The patient in case1 was infected but asymptomatic, whereas the patient in case 2 had pneumonia before surgery. COVIDSurg Collaborative [9] reported that for patients infected with COVID-19 before surgery, the 30day postoperative mortality rate of emergency surgery was higher than that of elective surgery. In addition, the mortality rate with pulmonary complications was $39.6 \%$ higher than $4.6 \%$ for patients without pulmonary complications. This report also shows predictors of mortality were consistent with male sex, age 70 years or older, American Society of Anesthesiologist grades III to V, emergency surgery, major surgery; therefore, it is important to consider that these predictors may worsen for acute appendicitis in patients with COVID-19 infection [9].

Moreover, steroid pulse therapy is used as a treatment for lung complications in COVID-19. Corticosteroids interfere with leukocyte and macrophage infiltration, reduce collagen synthesis and wound maturation, and interfere with the natural wound healing process [10]. In case 2 with pneumonia, steroid therapy was administered before and after surgery; wound dehiscence occurred on a postoperative day 23 . Therefore, it is necessary to observe the possibility of incomplete wound healing in patients on steroid treatment. Wound complications also increase when the patient is receiving ventilation support [11]. Risk factors for wound dehiscence include postoperative pneumonia, reintubation, and failure to wean [12]. Therefore, it is necessary to consider risk factors for wound complications in COVID-19 patients.

In conclusion, emergency surgery may be required even in the COVID-19 pandemic, and medical staff and related medical personnel must be aware of the considerations before, during, and after surgery. As diagnosis of acute appendicitis may be delayed with nonspecific symptoms and signs such as fever and leukocytosis, an image study should be considered to lower the risk of complications. Personal protective devices should be thoroughly implemented with negative pressure facilities. Surgeons should also consider the development of pneumonia and wound dehiscence in COVID-19 patients after surgery.

\section{CONFLICT OF INTEREST}

No potential conflict of interest relevant to this article was reported.

\section{REFERENCES}

1. World Health Organization (WHO). WHO announces COVID-19 outbreak a pandemic [Internet]. Geneva: WHO; 2020 [cited 2021 Jul 27]. Available from: https://www.euro.who.int/en/ health-topics/health-emergencies/coronavirus-covid-19/news/ news/2020/3/who-announces-covid-19-outbreak-a-pandemic.

2. Fagerström A, Paajanen P, Saarelainen H, Ahonen-Siirtola M, Ukkonen M, Miettinen P, et al. Non-specific abdominal pain remains as the most common reason for acute abdomen: 26-year retrospective audit in one emergency unit. Scand J Gastroenterol 2017;52:1072-7.

3. Yang Y, Li Y, Du X. Acute complex appendicitis during the COVID-19 epidemic: a single-institution retrospective analysis based on real-world data. Am J Emerg Med 2021;46:74-7.

4. Bajomo O, Hampal R, Sykes P, Miah A. Managing appendicitis during the COVID-19 era: a single centre experience \& implications for future practice. Ann Med Surg (Lond) 2021;63:102168.

5. Brown MA. Imaging acute appendicitis. Semin Ultrasound CT MR 2008;29:293-307.

6. Somers K, Abd Elwahab S, Raza MZ, O'Grady S, DeMarchi J, Butt A, et al. Impact of the COVID-19 pandemic on management and 
outcomes in acute appendicitis: should these new practices be the norm? Surgeon 2021;19:e310-7.

7. De Simone B, Chouillard E, Sartelli M, Biffl WL, Di Saverio S, Moore EE, et al. The management of surgical patients in the emergency setting during COVID-19 pandemic: the WSES position paper. World J Emerg Surg 2021;16:14.

8. Mowbray NG, Ansell J, Horwood J, Cornish J, Rizkallah P, Parker A, et al. Safe management of surgical smoke in the age of COVID-19. Br J Surg 2020;107:1406-13.

9. COVIDSurg Collaborative. Mortality and pulmonary complications in patients undergoing surgery with perioperative SARSCoV-2 infection: an international cohort study. Lancet 2020;396:
27-38.

10. Liu D, Ahmet A, Ward L, Krishnamoorthy P, Mandelcorn ED, Leigh $\mathrm{R}$, et al. A practical guide to the monitoring and management of the complications of systemic corticosteroid therapy. Allergy Asthma Clin Immunol 2013;9:30.

11. Sandy-Hodgetts K, Carville K, Leslie GD. Determining risk factors for surgical wound dehiscence: a literature review. Int Wound J 2015;12:265-75.

12. Webster C, Neumayer L, Smout R, Horn S, Daley J, Henderson W, et al. Prognostic models of abdominal wound dehiscence after laparotomy. J Surg Res 2003;109:130-7. 